\title{
Biosynthesis of 2-Deoxystreptamine-containing Antibiotics in Streptoalloteichus hindustanus JCM 3268: Characterization of 2-Deoxy-scyllo-inosose Synthase
}

\author{
Toshifumi Hirayama, Hideyuki Tamegai ${ }^{\dagger}$, Fumitaka Kudo, Kazumasa Kojima, \\ Katsumi Kakinuma, Tadashi Eguchi
}

Received: February 20, 2006 / Accepted: May 24, 2006

(C) Japan Antibiotics Research Association

\begin{abstract}
A part of the new biosynthetic gene cluster for 2-deoxystreptamine-containing antibiotics was identified from Streptoalloteichus hindustanus. The alloH gene in the gene cluster was deduced to encode 2-deoxy-scyllo-inosose synthase and the expressed protein AlloH was confirmed to have this enzyme activity. Furthermore, biochemical properties of AlloH were studied.
\end{abstract}

Keywords 2-deoxystreptamine biosynthetic genes, Streptoalloteichus hindustanus, 2-deoxy-scyllo-inosose synthase, kinetics

Aminoglycosides are an important group of antibiotics in clinical use for a long time. Even today, new activities of aminoglycosides are being discovered; for example, the activity of arginine complexes against $\operatorname{HIV}[1,2]$. Aminoglycosides are structurally oligosaccharides containing one of a small number of specific aminocyclitols and various amino sugars, which are connected together through the glycosidic bonds. Indeed, the biosynthetic enzymes

T. Eguchi (Corresponding author), T. Hirayama: Department of Chemistry and Materials Science, Tokyo Institute of Technology, 2-12-1 O-okayama, Meguro-ku, Tokyo 152-8551, Japan,

E-mail: eguchi@cms.titech.ac.jp

H. Tamegai, F. Kudo, K. Kojima, K. Kakinuma: Department of Chemistry, Tokyo Institute of Technology, 2-12-1 O-okayama, Meguro-ku, Tokyo 152-8551, Japan

$\dagger$ Present address: Department of Chemistry, College of Humanities and Sciences, Nihon University, 3-25-40 Sakurajosui, Setagaya-ku, Tokyo 156-8550, Japan catalyze the syntheses of each unit and the transfer reactions, which connect them. Therefore, structurally different aminoglycosides are expected to be constructed by combination of such biosynthetic enzymes through the gene organization in microorganisms. 2-Deoxy-scylloinosose (DOI) synthase catalyzes the first crucial carbocyclization in the most abundant aminocyclitol 2deoxystreptamine (DOS). Our identification of this gene in the butirosin producer Bacillus circulans [3], is an important key to the identification of many DOS-containing antibiotic gene clusters. So far, in addition to the butirosin biosynthetic gene cluster $[4,5]$, the biosynthetic genes for tobramycin [6], kanamycin [7, 8], neomycin [9, 10], ribostamycin [11], hygromycin B (only sequence data available; AJ628642), istamycin (AJ845083), lividomycin (AJ748832), paromomycin (AJ628955), and apramycin (AJ629123) from Streptomyces, and gentamicin [12, 13] from Micromonospora have been identified. Recombination of various these genes is expected to lead novel aminoglycosides by combinatorial biosynthesis.

We here report the identification of the biosynthetic gene cluster of DOS-containing antibiotics in Streptoalloteichus hindustanus [14], which, as well as Streptomyces tenebrarius, is known as an apramycin/tobramycin producer (Scheme 1). Streptoalloteichus hindustanus presents a new genus as a source of aminocyclitol biosynthetic genes beyond Streptomyces, Micoromonospora, and Bacillus, and thus it was anticipated to provide novel features of the biosynthetic enzymes.

Among the DOS-containing antibiotic biosynthetic gene clusters, the gene for L-glutamine:DOI aminotransferase is completely conserved and the enzyme catalyzes both transaminations in the DOS-formation [15]. This type of 


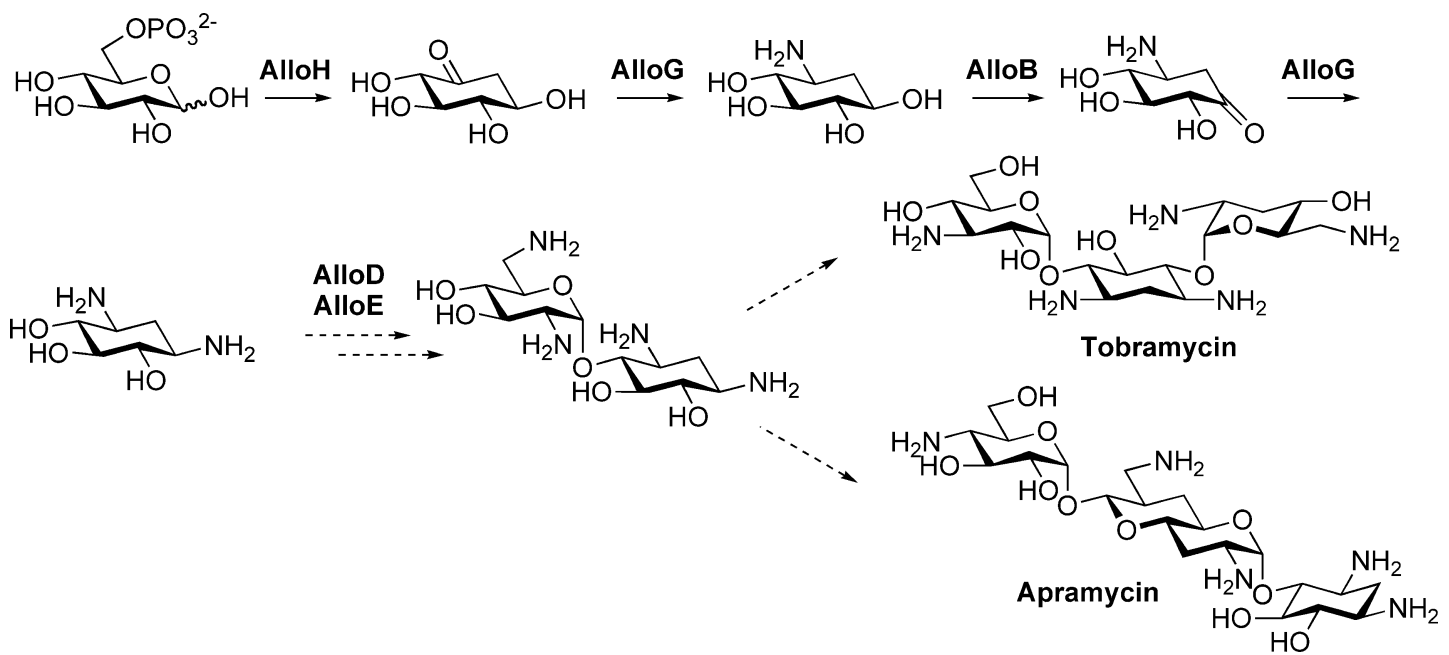

Scheme 1 Biosynthesis of 2-deoxystreptamine-containing antibiotics in Streptoalloteichus hindustanus.

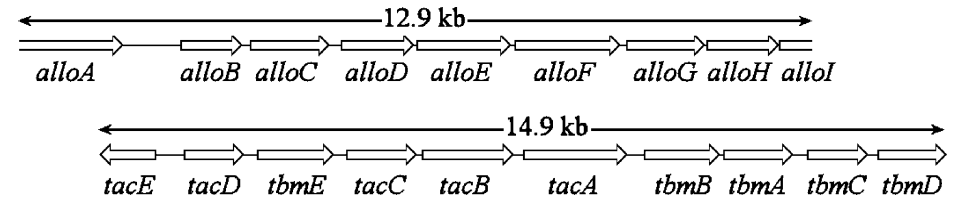

Fig. 1 Comparison of the DOS-containing antibiotic biosynthetic genes in Streptoalloteichus hindustanus (upper) and in Streptomyces tenebrarius (bottom).

The allo genes in Streptoalloteichus hindustanus, except for alloA, showed significant homology to the tobramycin biosynthetic genes in Streptomyces tenebrarius and the arrangement of these homologous genes is also the same. See also Table 1.

Table 1 Gene cluster from Streptoalloteichus hindustanus

\begin{tabular}{|c|c|c|c|c|}
\hline Genes & Size (bp) & Products of homologous genes & Origins & Homology \\
\hline alloA & 1687 & putative dehydrogenase & Bradyrhizobium japonicum & $36 \%$ \\
\hline alloB & 1020 & putative dehydrogenase (tacD) & Streptomyces tenebrarius & $79 \%$ \\
\hline alloC & 1299 & putative transport protein ( $t b m E$ ) & Streptomyces tenebrarius & $62 \%$ \\
\hline alloD & 1188 & putative aminotransferase $(\operatorname{tac} C)$ & Streptomyces tenebrarius & $81 \%$ \\
\hline alloE & 1527 & putative dehydrogenase (tacB) & Streptomyces tenebrarius & $78 \%$ \\
\hline alloF & 1713 & putative carbamoyltransferase (tacA) & Streptomyces tenebrarius & $87 \%$ \\
\hline alloG & 1275 & DOI aminotransferase $(t b m B)$ & Streptomyces tenebrarius & $87 \%$ \\
\hline alloH & 1158 & DOI synthase (tbmA) & Streptomyces tenebrarius & $81 \%$ \\
\hline allol & $>558$ & putative dehydrogenase $(t b m C)$ & Streptomyces tenebrarius & $69 \%$ \\
\hline
\end{tabular}

aminotransferase gene is also seen in the streptomycin [16] and fortimicin (AJ628421) biosynthetic gene clusters and was proposed to be a common gene among any aminocyclitol biosynthetic systems [16]. In fact, using the genetic information of this enzyme, many DOS-containing antibiotic biosynthetic gene clusters have been identified.

Similarly, by PCR screening against a cosmid library (using pOJ446 as a vector) of Sau3AI digested genomic
DNA of Streptoalloteichus hindustanus using the degenerate primers to amplify the aminotransferase gene [17], a cosmid callo16-16 was identified (Fig. 1 and Table 1). As a result of DNA sequence analysis, seven complete and two incomplete open reading frames (ORFs) were found in the gene cluster (deposited as AB103327) and, with the exception of alloA, showed significant homology to the tobramycin biosynthetic genes from Streptomyces 


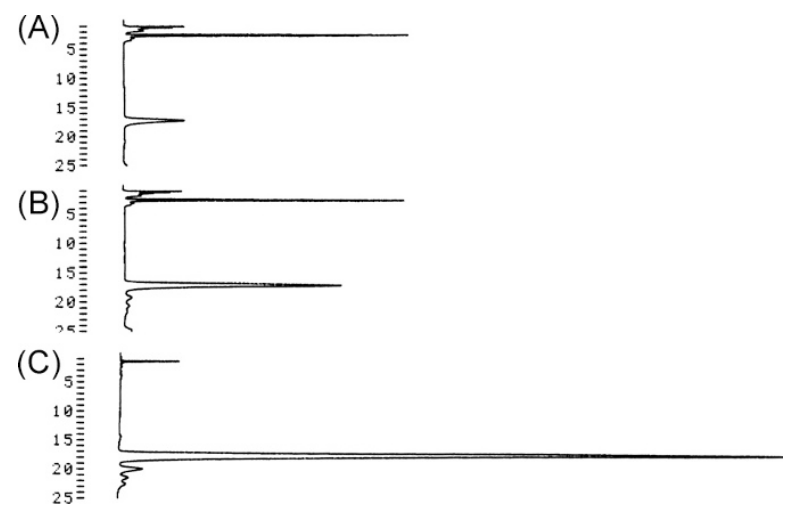

Fig. 2 HPLC of the derivatives from enzyme reaction using AlloH (A), (A)+authentic DOl oxime derivatives (B), authentic DOI oxime derivatives (C).

tenebrarius (Table 1). The arrangement of these homologous genes is also the same. Thus, the present identified gene cluster appears to be responsible to the tobramycin biosynthesis in Streptoalloteichus hindustanus. The alloA gene encodes a putative PQQ-dependent alcohol dehydrogenase that has not been seen in other aminoglycoside biosynthetic gene clusters, suggesting that possibly the alloA gene may be outside the biosynthetic cluster.

To confirm involvement of the allo genes in the antibiotic biosynthesis, the function of alloH gene encoding DOI synthase was investigated. Although several DOI synthases were heterologously expressed and found to be active, their biochemical properties have not been reported except for the single case of BtrC from B. circulans [3]. Since DOI is a versatile material for fine chemicals such as catechol [18] and carbaglucose [19], a highly efficient enzyme is desirable. Therefore, the enzymatic properties of AlloH were determined in the present study.

The alloH gene was thus expressed in Escherichia coli in a standard manner and the expressed AlloH was purified to homogeneity by DEAE Sepharose chromatography and gel filtration. Following the previously reported conditions for the BtrC reaction [3], AlloH was assayed with $5 \mathrm{mM}$ of glucose-6-phosphate and $5 \mathrm{mM}$ of $\mathrm{NAD}^{+}$at $46^{\circ} \mathrm{C}$ for 30 minutes. As shown in Fig. 2, AlloH was clearly confirmed as DOI synthase, indicating that the allo gene cluster is responsible for the biosynthesis of DOS-containing aminoglycoside antibiotics in Streptoalloteichus hindustanus.

Using the pure enzyme, the kinetic constants of AlloH were determined in a standard manner to be $0.075 \mathrm{~s}^{-1}$ and $0.69 \mathrm{mM}$ for $k_{\text {cat }}$ and $K_{\mathrm{m}}$ (for G-6-P), respectively (Table 2). Compared with those of the BtrC, $1.0 \mathrm{~s}^{-1}\left(k_{\text {cat }}\right)$ and $0.21 \mathrm{mM}\left(K_{\mathrm{m}}\right)$, AlloH was less efficient than BtrC. The $k_{\text {cat }}$
Table 2 Kinetic constants of AlloH and BtrC

\begin{tabular}{cccc}
\hline & $K_{\mathrm{m}}(\mathrm{mM})$ & $k_{\text {cat }}\left(\mathrm{sec}^{-1}\right)$ & $k_{\text {cat }} / K_{\mathrm{m}}\left(\mathrm{mM}^{-1} \mathrm{sec}^{-1}\right)$ \\
\hline AlloH & 0.69 & 0.075 & 0.11 \\
BtrC & 0.21 & 1.0 & 4.8 \\
\hline
\end{tabular}

The $K_{\mathrm{m}}$ values for G-6-P are shown.

value was significantly lower, indicating that the catalytic amino acid residues may not be well organized in the active site of AlloH. The probable catalytically important amino acids presumed from the crystal structure of 3dehydroquinate synthase [20], which catalyzes a similar reaction in the shikimate pathway, are highly conserved in AlloH and BtrC [3, 21]. No metal was required during purification of AlloH, whereas BtrC was inactive without $\mathrm{Co}^{2-}$ ion. In addition, an association with $\mathrm{NAD}^{+}$was observed in the reaction at low concentrations (data not shown). Although these properties may affect on the catalytic efficiency, the reason for the lower activity of AlloH is unclear at the moment. Amino acid sequences of all known DOI synthases from actinomycete are very similar but somewhat different to that of BtrC from Bacillus. This may suggest that all of the actinomycete DOI synthases are of lower activity. Detailed studies of DOI synthases including BtrC on the molecular level may open a way to engineer the enzymes to be more efficient.

In the present paper, a new aminoglycoside biosynthetic gene cluster from Streptoalloteichus hindustanus was identified showing that the allo genes are quite similar to the other known aminoglycosides biosynthetic genes from Streptomyces, Micromonospora, and Bacillus. This suggests that the biosynthetic genes were horizontally transferred during evolution and organized in each microorganism, and raises the likelihood that combinatorial fashioned arrangement of the genes beyond species could produce structurally diverse natural/unnatural aminoglycoside antibiotics. Indeed, the unknown functional genes in the biosynthetic gene clusters should be characterized for creation of made-to-order antibiotics.

While this manuscript was in preparation, a part of the apramycin biosynthetic gene cluster derived from Streptoalloteichus hindustanus DSM44523 (accession number AJ875019) was opened to public on the web. This cluster is different from the present identified cluster. Therefore, Streptoalloteichus hindustanus seems to have two sets of DOS-containing antibiotic biosynthetic gene cluster for tobramycin and apramycin. 
References

1. Litovchick A, Lapidot A, Eisenstein M, Kalinkovich A, Borkow G. Neomycin B-arginine conjugate, a novel HIV-1 Tat antagonist: synthesis and anti-HIV activities. Biochemistry 40: 15612-15623 (2001)

2. Lapidot A, Vijayabaskar V, Litovchick A, Yu J, James TL. Structure-activity relationships of aminoglycoside-arginine conjugates that bind HIV-1 RNAs as determined by fluorescence and NMR spectroscopy. FEBS Lett 577: 415-421 (2004)

3. Kudo F, Tamegai H, Fujiwara T, Tagami U, Hirayama K, Kakinuma K. Molecular cloning of the gene for the key carbocycle-forming enzyme in the biosynthesis of 2deoxystreptamine-containing aminocyclitol antibiotics and its comparison with dehydroquinate synthase. J Antibiot 52: 559-571 (1999)

4. Ota Y, Tamegai H, Kudo F, Kuriki H, Koike-Takeshita A, Eguchi T, Kakinuma K. Butirosin-biosynthetic gene cluster from Bacillus circulans. J Antibiot 53: 1158-1167 (2000)

5. Kudo F, Numakura M, Tamegai H, Yamamoto H, Eguchi T, Kakinuma K. Extended sequence and functional analysis of the butirosin biosynthetic gene cluster in Bacillus circulans SANK 72073. J Antibiot 58: 373-379 (2005)

6. Kharel MK, Basnet DB, Lee HC, Liou K, Woo JS, Kim BG, Sohng JK. Isolation and characterization of the tobramycin biosynthetic gene cluster from Streptomyces tenebrarius. FEMS Microbiol Lett 230: 185-190 (2004)

7. Yanai K, Murakami T. The kanamycin biosynthetic gene cluster from Streptomyces kanamyceticus. J Antibiot 57: 351-354 (2004)

8. Kharel MK, Subba B, Basnet DB, Woo JS, Lee HC, Liou K, Sohng JK. A gene cluster for biosynthesis of kanamycin from Streptomyces kanamyceticus: comparison with gentamicin biosynthetic gene cluster. Arch Biochem Biophys 429: 204-214 (2004)

9. Huang F, Haydock SF, Mironenko T, Spiteller D, Li Y, Spencer JB. The neomycin biosynthetic gene cluster of Streptomyces fradiae NCIMB 8233: characterisation of an aminotransferase involved in the formation of 2deoxystreptamine. Org Biomol Chem 3: 1410-1418 (2005)

10. Kudo F, Yamamoto Y, Yokoyama K, Eguchi T, Kakinuma K. Biosynthesis of 2-deoxystreptamine by three crucial enzymes in Streptomyces fradiae NBRC12773. J Antibiot 58: 766-774 (2005)

11. Subba B, Kharel MK, Lee HC, Liou K, Kim B-G, Sohng JK. The ribostamycin biosynthetic gene cluster in
Streptomyces ribosidificus: comparison with butirosin biosynthesis. Mol Cells 20: 90-96 (2005)

12. Unwin J, Standage S, Alexander D, Hosted T Jr, Horan AC, Wellington EMH. Gene cluster in Micromonospora echinospora ATCC15835 for the biosynthesis of the gentamicin C complex. J Antibiot 57: 436-445 (2004)

13. Kharel MK, Basnet DB, Lee HC, Liou K, Moon YH, Kim JJ, Woo JS, Sohng JK. Molecular cloning and characterization of a 2-deoxystreptamine biosynthetic gene cluster in gentamicin-producing Micromonospora echinospora ATCC15835. Mol Cells 18: 71-78 (2004)

14. Tomita K, Uenoyama Y, Numata K, Sasahira T, Hoshino Y, Fujisawa K, Tsukiura H, Kawaguchi H. Streptoalloteichus, a new genus of the family Actinoplanaceae. J Antibiot 31: 497-510 (1978)

15. Yokoyama K, Kudo F, Kuwahara M, Inomata K, Tamegai H, Eguchi T, Kakinuma K. Stereochemical recognition of doubly functional aminotransferase in 2-deoxystreptamine biosynthesis. J Am Chem Soc 127: 5869-5874 (2005)

16. Ahlert J, Distler J, Mansouri K, Piepersberg W. Identification of stsC, the gene encoding the L-glutamine: scyllo-inosose aminotransferase from streptomycin-producing Streptomycetes. Arch Microbiol 168: 102-113 (1997)

17. Tamegai H, Eguchi T, Kakinuma K. First identification of Streptomyces genes involved in the biosynthesis of 2deoxystreptamine-containing aminoglycoside antibioticsgenetic and evolutionary analysis of L-glutamine: 2-deoxyscyllo-inosose aminotransferase genes. J Antibiot 55: 1016-1018 (2002)

18. Kakinuma K, Nango E, Kudo F, Matsushima Y, Eguchi T. An expeditious chemo-enzymatic route from glucose to catechol by the use of 2-deoxy-scyllo-inosose synthase. Tetrahedron Lett 41: 1935-1938 (2000)

19. Nango E, Eguchi T, Kakinuma K. Active site mapping of 2deoxy-scyllo-inosose synthase, the key starter enzyme for the biosynthesis of 2-deoxystreptamine. Mechanism-based inhibition and identification of lysine-141 as the entrapped nucleophile. J Org Chem 69: 593-600 (2004)

20. Carpenter EP, Hawkins AR, Frost JW, Brown KA. Structure of dehydroquinate synthase reveals an active site capable of multistep catalysis. Nature 394: 299-302 (1998)

21. Eguchi T, Sasaki S, Huang Z, Kakinuma K. Importance of specific hydrogen-bond donor-acceptor interactions for the key carbocycle-forming reaction catalyzed by 2-deoxyscyllo-inosose synthase in the biosynthesis of 2deoxystreptamine-containing aminocyclitol antibiotics. J Org Chem 67: 3979-3984 (2002) 